\title{
Cognitive Behavior Therapy for Psychogenic Cough in Children : a case series
}

\author{
Shahzadi Malhotra ${ }^{1}$, Shweta Tandon ${ }^{2}$ \\ ${ }^{1,2}$ Assistant Professor, Department of Clinical Psychology, Chacha Nehru Bal Chikitsalaya, Delhi. \\ Corresponding author: Shahzadi Malhotra \\ Email -shahzadimalhotra1@gmail.com
}

\begin{abstract}
Introduction: Cough is the most common presenting symptom in pediatric primary care settings. Cough can impact a child's activity level and ability to sleep, play or attend school and is often a source of parental anxiety. However, an etiology of cough is not always easily identified even after a thorough systematic investigation and psychological and neurological conditions are in the differential diagnosis. Psychogenic cough is diagnosed in cases without a clear pulmonary or extrapulmonary etiology in the presence of some suggestive clinical characteristics and/or an association with psychological issue. Psychogenic cough has been reported to be the second most common cause of chronic cough in children of age 6-16 years.

Methodology: The present paper highlights four case reports of children who presented with symptoms of psychogenic cough in the OPD of CNBC hospital, Delhi.The presenting nature of the symptoms along with the underlying psychological factors have been discussed. Cognitive Behavioural techniques like distraction and cognitive restructuring interventions with the children along with family interventions were used.

Conclusions: All the children showed significant improvement at the end of therapy. Three month follow up showed that the results were maintained. Cognitive Behavioural approaches are found to be effective in managing psychogenic cough and the present paper highlights the process of cognitive behavioural management of psychogenic cough in children.
\end{abstract}

Key words: cognitive behavior therapy, cough, psychogenic cough, children.

(Paper received $-26^{\text {th }}$ February 2018, Peer review completed $-20^{\text {th }}$ March 2018)

(Accepted $-24^{\text {th }}$ March 2018)

\section{INTRODUCTION}

An automatic cough is a vagal sensory reflex occurring when irritant receptors located in the larynx, trachea, or large bronchi are stimulated [1,2]. Cough has been classified into various types depending upon its genesis. For instance, cough is classified as a tic disorder which is defined as sudden, quick, involuntary, and frequently repeated movements of a physiological group of muscles that serve no apparent purpose [2-4]. Psychogenic or barking cough is defined as a respiratory tic.

This nervous laryngeal cough or cry is emitted on an expiratory spasmodic contraction of the glottic adductors, repeated at frequent intervals during the day and generally ceasing during sleep. It consists of a short sharp, usually grating explosive cough that resembles the bark of a dog or the squeal of a puppy and as a rule can be resisted only for a time at the expense of painful emotion. There is no expectoration, no subsequent breathlessness or fatigue, and no change in the voice. Spraying the larynx with a topical anaesthetic does not seem to affect the condition. The patient claims to be unable to repeat the cough when requested to do so. Physical examination will usually be unremarkable [4]. 
Involuntary cough without an identified underlying organic reason has been repeatedly described. Psychogenic or "nervous" cough has generally been viewed as a clinical oddity and largely ignored by specialists text-books of medicine, psychiatry and otorhinolaryngology [5-7]. The association of respiration and emotions is well known. Clinicians are familiar with a wide assortment of respiratory aberrancies like breath holding spells in angry children, sighing respiration during states of grief, increased ventilation during anxiety or excitation, crying and sobbing, deep hyperventilation in hysteria, and precipitation of asthma attacks by emotion $[8,9]$.

A central feature in differentiating psychogenic from ordinary coughing is its unusual loudness [9]. It is usually croupy, barky, and explosive and disappears during sleep. It is resistant to antitussive medications. A typical "chin on chest" posture with the hand held to the neck while coughing may be noticed in some of the patients. The physical examination of the respiratory system as well as routine investigation do not reveal any relevant abnormality. Various factors causing psychogenic cough have been proposed. For example, it may be precipitated in children by maternal dominance [13], school phobia [14,15], social distress and withdrawal [16], dissatisfaction in family [17-20], an expression of hostility or selfpunishment, an upward displacement of genital conflicts, a plea for assorted secondary gains, conflicts over closeness, fear of rejection, and need for attention. The respiratory system provides an affective outlet through a conditioned somatic pathway of discharge.

One of the earliest detailed case reports of psychogenic cough accompanied by 1 year of follow-up was published by Bernstein [5] . He described a 12-year-old girl with what he named "the barking cough of puberty." Two months later, Baker [16] introduced the terms "chronic cough from habit or psychosomatic disease" in the differential diagnosis for chronic cough in children. The actual term "habit cough" was first used by Berman in 1966 [7]. He reported six children in whom he "relied solely on the art of suggestion" to produce the cessation of cough. His 2-year follow-up supported the success of his therapy. Since then, several descriptive terms have been used for this condition, for example, "functional or psychogenic cough" [10] , "psychogenic cough tic" [8], "operant cough" [9] , "honking" [11] , and "involuntary cough syndrome" [12] .

The different names given to this kind of cough include Psychogenic cough tic [1], Nervous cough [7], Tussis nervosa [21], Habit cough [12], Operant cough [22], Barking cough of puberty [13], Honking [11], The bark of hysteria [23], Expiratory tic [13], and Dora's cough [24].

Since there is paucity of literature on psychogenic cough, the present case series in discussed to highlight the genesis, maintenance and management of psychogenic cough in children.

\section{METHODOLOGY}

The present study was conducted in a tertiary care teaching hospital. The patients were screened by the otorhinolaryngologist and then referred to the Clinical Psychology outpatient department. All the patients were subjected to a detailed history (on a semi-structured proforma), physical examination, and mental status examination. The relevant blood and radiological investigations were done wherever required to assess physical status. Examination of the throat, nose, and ear was done in every case to rule out the organic disease. The precipitating or exacerbating factors were also recorded. Sociodemographic and clinical details along with precipitating factors of psychogenic cough were analyzed. After the diagnosis all the patients underwent psychotherapy sessions.

\section{Case 1}

The patient was a 5-year-old male child. Four months before he developed habit cough, the patient manifested severe coughing after contracting influenza. At that time, he was diagnosed with a pneumonia, and treated with intravenous antibiotics. A fortnight after his treatment for pnuemonia was completed, he developed a loud, harsh cough that occurred every other second for hours at a time, while awake. The cough ceased as soon as he fell asleep. 


\section{Case 2}

A 12-year-old female was brought with the complaints of chronic cough, which had begun 5 months ago. She had primary education, currently discontinued with her studies and was at home. She had no history of hospitalization before this problem. Her coughing was significantly affecting her life and social relationships. The coughing occurred daily, without sputum production or fever, rhinorrhea, or chest pain. She felt better when she had a distraction and cough persisted during the times of wakefulness. Cough typically was not present during sleep. There has been no recent exposure to tobacco smoke, a person with a chronic productive cough.

She has been hospitalized several times to treat this problem and investigations like CXR, CT spiral were normal. There was no abnormality found on any of the routine diagnostic procedures. She had normal sleep duration. There was no associated difficulty in swallowing or voice change. Patient was given trial of antibiotic therapy but with no success. Consultation with pediatrician and ENT specialist was done and the organicity was ruled out. There was no objective laboratory or radiologic evidence of any physical disease.

\section{Case 3}

Ms. R, a 10-year-old girl from rural background was brought by her maternal aunt for complaints of continuous coughing for last fifteen days. The patient was reportedly asymptomatic three weeks before her visit to the hospital when she came from her village to Delhi to stay with her aunt to join new school. Patient reported that she herself was willing to join new school. However, two days before her classes commenced she started having continuous coughing owing to which she could not join school. She could however, manage doing household chores without much difficulty.

There was no associated sputum, nasal discharge, lacrimation or cough. Local nasal examination, sinuscopy, X-ray chest and CT scan were normal. The coughing was continuous but did not occur during sleep. There was past history of two similar episodes as reported by the patient herself- both times when she took admission in school but had to drop because of coughing. There was no past history of allergy, breathlessness or any other significant physical illness. Before being referred to clinical psychologist for evaluation, the patient was given a trial of antihistaminics, steroids and bronchodilators but without any improvement. The child was referred for psychological evaluation. All the medications were gradually tapered off and detailed psychological evaluation was done.

\section{Case 4}

Master A, a 8 year old male child was brought by his mother to the OPD with chief complaints of cough with a peculiar sound since last 01 week. On exploration it ws revealed that the cough exacerbates early morning after getting up on weekdays. No such episodes of cough were observed on weekend. Further no coughing was observed during sleep, palytime. Mother reported that severity would minimize during T.V watching. There was no associated sputum, nasal discharge, lacrimation. Physical examination by the pediatrician and ENT specialist reported local throat, nasal examination, sinuscopy, X-ray chest to be normal. Child lives in a nuclear family and has one elder sister. Child reportedly shared cordial relationship with his sister.

\section{MANAGEMENT}

The treatment programme took place on one hour a week for six consecutive weeks. Each session was conducted by one therapist. The individual sessions used simple behavioural techniques before moving on to cognitive behaviour therapy.

Following techniques were used for psychotherapeutic management:

1. Psychoeducation and feelings identification-The first session focused on psychoeducation about anxiety and functional problems. Cognitive, somatic, and behavioral symptoms of anxiety were reviewed with emphasis on the association and interaction between somatic symptoms and anxiety, such as how anxiety and cough can exacerbate one another. For example, the therapist explained to the children that they have been refferred by their pediatrician because no physical 
cause of their cough has been established. They were explained that although their doctor was not able to locate a cause for their cough on any medical tests, the therapist understands that they are painful and distressing for the child and are getting in the way of his/her life. It was discussed with the children that how because of their cough, they were having trouble attending and enjoying school and social activities. It was also discussed that although the therapist, would not be able to exactly identify what comes first- whether child gets anxious and then cough starts or he/she has cough and then because of cough feels anxious but would still, however, could understand that the discomfort in body can make them feel more anxious and feeling anxious can make the body hurt more. In the initial sessions it was also explained that the children will learn ways to help your body feel better and help them feel less worried, because both these symptoms can go together." The session continued with instruction on identifying and communicating feelings. The session concluded with the identification of specific situations that induce anxiety and cough.

2. Relaxation and breathing retraining-The second session focused on children's physical symptoms. The specifics of children's bodily discomfort (i.e., the specific sensations and symptoms, severity, frequency, duration, situational antecedents) were explored. Simple diaphragmatic breathing exercises were also taught. During this session a 0-to-10 point scale is introduced to assign a quantitative measure of anxiety and cough. The child was asked to monitor and record his or her anxiety and physical pain daily to enhance early detection as well as to reveal possible interactions between cough and anxiety.

3. Behavioural Strategies - Behavioral strategies such as activity scheduling were introduced, in order to maximize mastery and pleasure. Distraction was also introduced and appropriate distractors were identified for each individual child.

4. Cognitive restructuring-The third and fourth sessions aimed to enhance realistic thinking in children. The therapist introduced the relation between thoughts and feelings, facilitating the children to identify maladaptive thoughts, and provided guidance in challenging negative expectations associated with cough and anxiety-producing situations. Wherever need aroused, children were given ample opportunity to ventilate their emotions. They were also given training in enhancing coping skills, especially on problem focused ways of coping. Use of coping self statements was also introduced order to help them deal effectively in some of the situations that led to negative emotions but in which techniques like distraction could not be used.

\section{Parent Sessions}

Most of the parents had a limited understanding of the symptoms and impairment associated with anxiety, ways in which their child's cough symptoms were related to anxiety, and parental behaviors that can inadvertently maintain anxiety and cough. Therefore, three parent sessions were developed to specifically address ways parents can help children manage cough and anxiety symptoms.

1. Parent Session 1-The initial parent session was scheduled at the outset of therapy with vthe child and followed immediately after history taking session. Parents were educated about the symptoms and how these symptoms are maintained by anxiety, and the connection between cough and anxiety. During the session, the therapist explored the details regarding the child's anxiety and family behaviors that might be addressed in future sessions. The course of psychotherapy and prognosis along with the need for following the therapy techniques regularly.

2. Parent Session 2 and 3- The therapist discussed parental responses to anxiety and cough (e.g., allowing child to stay home from school because of cough, giving undue attention) that may maintain symptoms. Parents were encouraged to discontinue behaviors that might maintain cough and anxiety symptoms (e.g., allowing avoidance, overprotection) and are taught specific skills such as rewarding nonanxious behaviors, ignoring unwanted behaviors, encouraging independence, and modeling nonanxious coping behaviors.

3. Parent sessions also focussed on helping parents carry out and monitor the proper implementation of techniques between sessions. Parents often find it difficult to facilitate exposures due to emotional distress and cough that children experienced. When exposures lead to increases in 
cough, it can be especially hard for families to comply with recommended out-of-session exercises. To avoid such non compliance, parents were explained extinction burst and were prepared on how to deal with such situations.

4. Parents were also explained the need to use extinction techniques consistently and to normalise child's daily routine to premorbid levels. Through the use of extinction, the secondary gains were gradually withdrawn.

\section{Treatment review and relapse prevention}

In the final session, the therapist and family reviewed skills learned, identified potential warning signs of relapse, and discussed strategies for relapse prevention.

\section{Outcome and Discussion}

All the four case reports discussed above fulfilled the criteria for psychogenic cough. All the physical examinations and investigations were normal and psychogenic stressors along with maintaining factors were recognized as contributing to their symptoms. Once the psychogenic nature of symptoms, their onset (due to underlying stress) and their perpetuation (due to secondary gains) were established, structured psychotherapy was given. There was a significant improvement followed by remission of cough. After three months of treatment all the four children reported almost negligible coughing and also their anxiety symptoms had remitted. It must be emphasized that treating physicians must consider psychogenic cough as one of the differential diagnosis when no physical cause can be established especially for children to avoid an unnecessary extensive medical evaluation and unneeded medications, parental anxiety and effect on school performance and social functioning.

\section{REFERENCES}

1. Cohlan SQ, Stone SM. The cough and the bedsheet. Pediatrics 1984;74:11-15.

2. Brescta MA. Tics in childhood. Arch Pediatr 1938;55:703.

3. Kardos P. Proposals for a rationale and for rational diagnosis of coughs. Pneumologie 2000;54:110-115.

4. Grumet GW. Psychogenic coughing: A review and case report. Compr Psychiatry 1987;28:28-34.

5. Bernstein L. A respiratory tic: "the barking cough of puberty." Report of a case treated success-fully. Laryngoscope 1963;73:315-9.

6. $\quad$ Baker Jr DC. Chronic cough in children. N Y State J Med 1963;63:1535-9.

7. Berman BA. Habit cough in adolescent children. Ann Allergy 1966;24:43-6. 4.

8. Kravitz H, Gomberg RM, Burnstine RC, Hagler S, Korach A. Psychogenic cough tic in children and adolescents. Nine case histories illustrate the need for re-evaluation of this common but frequently unrecognized problem. Clin Pediatr (Phila) 1969;8:580-3.

9. Munford PR, Liberman RP. Differential attention in the treatment of operant cough. J Behav Med 1978;1:289-95.

10. Rabin CB. Disturbances of respiration of functional origin. J Asthma Res 1968;5:295-308.

11. Weinberg EG. 'Honking': Psychogenic cough tic in children. S Afr Med J 1980;57:198-200.

12. Rojas AR, Sachs MI, Yunginger JW, O'Connell EJ. Childhood involuntary cough syndrome: a long-term follow-up study. Ann Allergy 1991;66:106.

13. Bernstein IA. A respiratory tic: "The barking cough of puberty.” Laryngoscope 1963; 73:315-319.

14. Kravitz H, Gomberg RM, Burnstine RC, Hagler S, Korach A. Psychogenic cough tic in "children and adolescents.' Clin Pediatr 1969;8:580-583.

15. Shuper A, Mukamel M, Mimouni M, Lerman M, Varsano L. Psychogenic cough. Arch Dis Child 1983;58:745-747.

16. Mamford PR, Reardson D, Liberman RP, Allen L. Behavioural treatment of hysterical coughing and mutism: A case study. J Consult Clin Psychol 1976;44:1008-1014.

17. Lindenbaum S, Clark D. Toward an integrative approach to psychotherapy with children. Am J Orthopsychiatry 1983;53:449-459.

18. Erenberg G. Psychogenic cough. Pediatrics 2001;108:819-820.

19. Riegel B. Warmath JE, Middaugh SJ, Kee WG, Nicholson LC, Melton DM, Parikh DK, Rosenberg JC. Psychogenic cough treated with biofeedback and psychotherapy. A review and case report. Am J Phys Med Rehabil 1995;74:155-158.

20. Malhotra S, Chakrabarti S. Psychogenic cough-A case report. Indian J Med Sci 1999;53:127-129.

21. Breuer J, Freud S. Studies on hysteria. In: Standard edition of the complete psychological works of Sigmund Freud, Vol. 2. London: Hogarth Press; 1955. 
22. Manford PR, Libermann RP. Differential attention in the treatment of operant cough. J Behav Med 1978;1:289-295.

23. Thompson ES. Cough. In: Quoin R, ed., A dictionary of medicine. London: Longmans Green; 1884.

24. Freud S. Fragment of an analysis of a case of hysteria. In: The second edition of the complete psychological works. London: Hogarth; 1953.

Acknowledgements - Nil;

Conflict of Interest - Nil;

Funding - Nil. 